\title{
Effects of taxol resistance gene 1 expression on the chemosensitivity of SGC-7901 cells to oxaliplatin
}

\author{
LIANCHENG LIU $^{1}$, ZHIGANG BAI ${ }^{1,2}$, XUEMEI MA ${ }^{1,2}$, TINGTING WANG $^{1,2}$, \\ $\mathrm{YAO}_{\mathrm{YANG}^{1,2}}$ and ZHONGTAO $\mathrm{ZHANG}^{1,2}$ \\ ${ }^{1}$ Department of General Surgery, Beijing Friendship Hospital, Capital Medical University; \\ ${ }^{2}$ Beijing Key Laboratory of Cancer Invasion and Metastasis Research, \\ National Clinical Research Center for Digestive Diseases, \\ Beijing Friendship Hospital, Beijing 100050, P.R. China
}

Received April 9, 2015; Accepted November 25, 2015

DOI: $10.3892 /$ etm.2016.2994

\begin{abstract}
The present study aimed to evaluate the role of taxol resistance gene 1 (Txr1) in the development of oxaliplatin (L-OHP) resistance in gastric cancer (GC). Using SGC-7901 cells as a model, Txr1 was exogenously expressed or knocked down using small interfering RNA. Quantitative polymerase chain reaction (qPCR) and western blotting were performed to establish whether the Txrl gene is involved in chemoresistance, and cell proliferation was assessed using an MTS assay. To this end, the mRNA and protein levels of Txr1, thrombospondin-1 and excision repair cross-complementing 1 protein were measured using qPCR and western blotting, respectively. Txr1-knockdown significantly increased the sensitivity of the SGC-7901 cells to L-OHP, whereas Txr1 overexpression promoted the resistance of the SGC-7901 cells to L-OHP. Exogenous Txr1 expression in the SGC-7901 cells induced L-OHP resistance, and the siRNA knockdown of Txr1 sensitized the human GC cells to L-OHP. Txr1 is, therefore, likely to play a role in L-OHP resistance, acting via TSP1, and should be investigated as a potential therapeutic target in the treatment of GC.
\end{abstract}

\section{Introduction}

Gastric cancer (GC) is the third leading cause of cancer-associated mortality across the two genders, and the fifth most common malignancy worldwide. Half of the total GC cases worldwide occur in Eastern Asia, mainly in China (1), and the management of the disease continues to evolve. In Asia, an extended resection followed by adjuvant chemotherapy represents the standard care

Correspondence to: Dr Zhongtao Zhang, Department of General Surgery, Beijing Friendship Hospital, Capital Medical University, 95 Yongan Road, Beijing 100050, P.R. China

E-mail: zhangzht@medmail.com.cn

Key words: Txrl, gastric cancer, oxaliplatin, resistance regimen (2). Oxaliplatin (L-OHP) forms the basis of treatment of multiple types of cancer, and the current GC adjuvant therapy regimen in Eastern Asia is S-1 or capecitabine combined with L-OHP $(2,3)$. Although L-OHP responsiveness is initially high, patients ultimately develop L-OHP resistance (4). Nevertheless, the exact mechanisms of L-OHP resistance in GC cells have not been elucidated. Strategies to decrease the resistance of GC to platinum-based chemotherapy must, therefore, be developed.

DNA repair capability is a key contributor to the resistance to L-OHP; specifically, the excision repair cross-complementation group 1 (ERCC1) protein has an essential role in nucleotide excision repair (5). ERCC1 may be an effective prognostic factor for gastric cancer patients following radical resection; it has previously been suggested that ERCC1-negative patients may benefit more from adjuvant chemotherapy (6). Furthermore, high ERCC1 expression may be a critical indicator of a poor clinical outcome, and may be predictive of resistance to pharmaceutical treatment in advanced GC patients (7). Although L-OHP resistance may be partially explained by ERCC1 expression, the effects of other L-OHP resistance-associated genes, including taxol resistance gene 1 (Txr1), should also be evaluated.

Txr1 is a drug resistance gene receiving increasing attention; this gene decreases thrombospondin-1 (TSP1) secretion and causes taxol resistance (8). Previous studies using lung (9) and breast (10) cancer cells have revealed that Txr1 upregulation may induce drug resistance in cancer cells. Our previous study revealed that Txr1 expression is also associated with the 5-year overall survival (OS) rate of GC patients and may represent a target to reverse L-OHP resistance in GC (11).

The aim of the present study was to identify the molecular mechanism of Txr1-associated L-OHP resistance. A variety of proteins, including ERCC1 and TSP1, were detected; these proteins may have clinical potential as predictive biomarkers.

\section{Materials and methods}

Cell culture and treatments. The human GC cell lines, AGS, SGC-7901, MNK-45 and BGC-823, were purchased from China Infrastructure of Cell Line Resources (Beijing, China) 
and subsequently cultured in Gibco Dulbecco's modified Eagle's medium supplemented with $10 \%$ heat-inactivated fetal calf serum, $100 \mathrm{U} / \mathrm{ml}$ penicillin and $100 \mu \mathrm{g} / \mathrm{ml}$ streptomycin (Thermo Fisher Scientific, Inc., Waltham, MA, USA). The cells were cultured in a $5 \% \mathrm{CO}_{2}$, humidified incubator at $37^{\circ} \mathrm{C}$. Goat polyclonal antibodies against Txr1 (1:100; cat. no., sc-244548), and mouse monoclonal antibodies against $\beta$-actin $(1: 1,000$; cat. no., sc-8432) and TSP1 (1:100; cat. no., sc-59887) were acquired from Santa Cruz Biotechnology, Inc. (Dallas, TX, USA). The mouse anti-ERCC1 monoclonal antibody (1:500; cat. no., MA5-13830) was purchased from Thermo Fisher Scientific, Inc.

Quantitative polymerase chain reaction ( $q P C R)$. Total RNA from cell line samples was prepared using TRIzol reagent (Thermo Fisher Scientific, Inc.) in accordance with the manufacturer's protocols. RNA was diluted to $100 \mathrm{ng} / \mu \mathrm{l}$ using DNase/RNase-free water and the quality and quantity of RNA was assessed using a NanoDrop ND-1000 spectrophotometer (Thermo Fisher Scientific, Inc., Wilmington, DE, USA), and the RNA samples were subsequently stored at $-80^{\circ} \mathrm{C}$. Complementary DNA synthesis was performed with reverse transcriptase using the Reverse Transcription system (cat. no., A3500; Promega Corporation, Madison, WI, USA) in accordance with the manufacturer's protocols. Total RNA (10 ng/ $/ \mathrm{l})$ in $5 \mu 1$ nuclease-free water was added to $3 \mu \mathrm{l} 5 \mathrm{X}$ RT primer, $1.5 \mu 110 \mathrm{X}$ reverse transcriptase buffer, $0.15 \mu 1100 \mathrm{mM}$ dNTPs, $0.19 \mu \mathrm{l}$ RNase inhibitor, $4.16 \mu \mathrm{l}$ nuclease-free water and 50 units reverse transcriptase in a total volume of $20 \mu \mathrm{l}$. The reaction was performed for $60 \mathrm{~min}$ at $42^{\circ} \mathrm{C}$, then for $5 \mathrm{~min}$ at $95^{\circ} \mathrm{C}$, in triplicate.

mRNA levels were quantified using a SYBR Green Real-Time PCR Master mix (Thermo Fisher Scientific, Inc.) and a 7500 Real-Time PCR system (Applied Biosystems; Thermo Fisher Scientific, Inc.). Primers to amplify the cDNA were obtained from Sangon Biotech Co., Ltd. (Shanghai, China). Thermal cycling was performed as follows: $95^{\circ} \mathrm{C}$ for $5 \mathrm{~min}$, followed by 38 cycles of $95^{\circ} \mathrm{C}$ for $15 \mathrm{sec}$ and $60^{\circ} \mathrm{C}$ for $60 \mathrm{sec}$. Relative expression was calculated using the comparative cycle quantification $\left(\mathrm{C}_{\mathrm{q}}\right)$ method $(\Delta \Delta \mathrm{Cq})$, and $\beta$-actin was used for normalization. All PCR amplifications were performed in triplicate and the experiment was repeated three times. The primers used are listed in Table I.

Lentivirus-mediated RNA interference and overexpression. Human Txr1 cDNA was subcloned into the plasmid pLenti6/V5. Txr1 and an empty control recombinant lentivirus (Lv.Txr1 and Lv.Empty) were generated by Shanghai GenePharma Co., Ltd. (Shanghai, China). A lentivirus expressing Txr1 small interfering (si)RNA (siTxrl) and a negative control lentivirus (siCON) were also generated by Shanghai GenePharma Co. Ltd.

Cell proliferation assay. Cell proliferation was analyzed in triplicate using the CellTiter 96 AQueous One Solution Cell Proliferation assay (MTS assay; Promega Corporation) in accordance with the manufacturer's protocols. Briefly, 5,000 cells/well were grown in RPMI-1640 medium (100 $\mu \mathrm{l}$ /well; Gibco; Thermo Fisher Scientific, Inc.) in 96-well plates and exposed to various concentrations of the experimental

Table I. Primers used to detect Txr1, TSP1 and ERCC1 expression by quantitative PCR.

Primer Sequence (5'-3')

Txr1

Forward

Reverse

TSP1

Forward

Reverse

ERCC1

Forward

Reverse

Txr1, taxol resistance gene 1; TSP1, thrombospondin-1; ERCC1, excision repair cross-complementing 1 protein.

drug $(0.001,0.01,0.1,1,10,100$ and 1,000 $\mu \mathrm{g} / \mathrm{ml})$. After $48 \mathrm{~h}$ of drug exposure, $20 \mu \mathrm{l}$ MTS was added to the medium and the cells were incubated in a $5 \% \mathrm{CO}_{2}$ incubator at $37^{\circ} \mathrm{C}$ for $2-4 \mathrm{~h}$. The absorbance, which is directly proportional to the number of viable cells in culture, was measured at $490 \mathrm{~nm}$ using a plate reader (Model 680; Bio-Rad Laboratories, Hercules, CA, USA). Relative growth was, therefore, calculated using the following formula: Growth $(\%)=\left[\left(\mathrm{A}_{\text {treated }}-\mathrm{A}_{\text {zero }}\right) /\left(\mathrm{A}_{\text {control }}-\mathrm{A}_{\text {zero }}\right)\right] \times 100$, where ' $A_{\text {treated }}$ ' was the number of living cells in the treated wells, ' $\mathrm{A}_{\text {control }}$ ' was the number of living cells in the untreated wells and ' $A_{\text {zero }}$ ' was the background absorbance, which was subtracted to correct for error.

Western blot analysis. Whole-cell extracts were prepared using a keyGEN Whole Cell Lysis assay (Nanjing KeyGen Biotech Co., Ltd., Nanjing, China). The samples were denatured by boiling for 5 min in loading buffer, separated using a $10 \%$ sodium dodecyl sulfate-polyacrylamide gel electrophoresis and electroblotted onto a polyvinylidene difluoride membrane (Thermo Fisher Scientific, Inc.). The membranes were incubated with primary antibodies for $2 \mathrm{~h}$, washed with Tris-buffered saline plus Tween 20 (TBST; Bio-Rad Laboratories, Inc.) buffer for $30 \mathrm{~min}$ and incubated with horseradish peroxidase-conjugated bovine anti-mouse $\mathrm{IgG}$ (1:10,000; cat. no., sc-2371) and chicken anti-goat immunoglobulin (Ig)G (1:10,000; cat. no., sc-2953) secondary antibodies at room temperature for $1 \mathrm{~h}$. The membranes were subsequently washed again with TBST for $30 \mathrm{~min}$, and the immunolabeled bands were detected using an enhanced chemiluminescence kit (SuperSignal West Pico Chemiluminescent Substrate kit; Thermo Fisher Scientific, Inc.). The blots were scanned using an Epson Perfection Photo Scanner (Tokyo, Japan) and analysis of these was performed with ImageJ (National Institutes of Health, Bethesda, MA, USA) by measuring the densities of the immunoreactive bands.

Flow cytometry (FCM) analysis. An Annexin V/propidium iodide (PI) double staining assay was performed using an Annexin V-fluorescein isothiocyanate apoptosis detection kit (Sigma-Aldrich, St. Louis, MO, USA) in accordance with the 
A

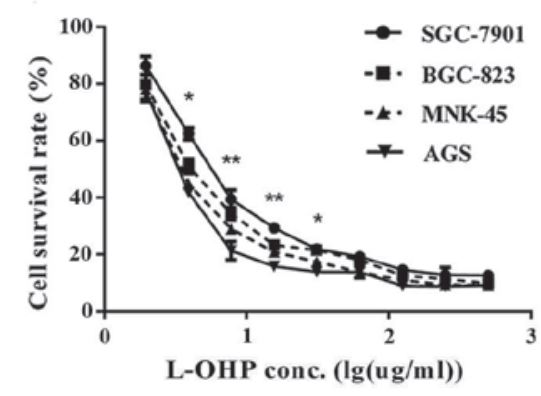

B

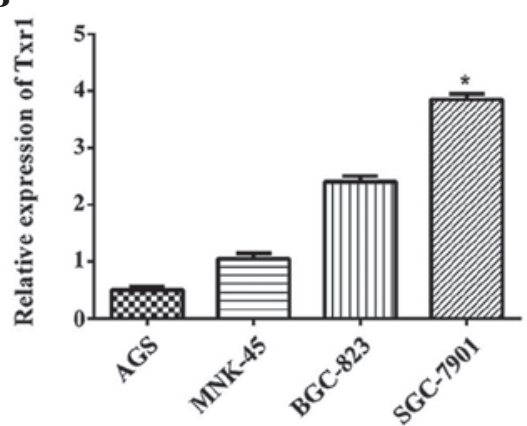

C

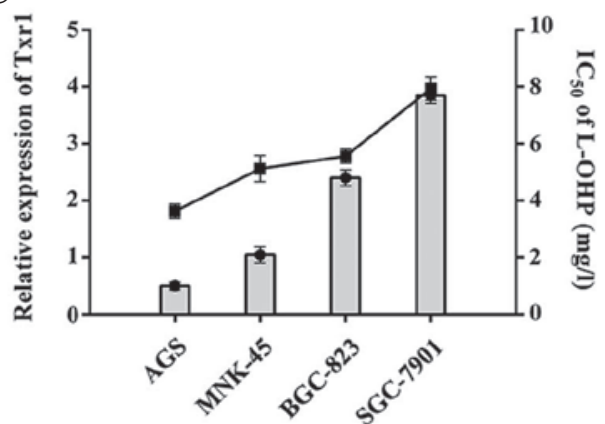

Figure 1. L-OHP sensitivity of GC cells is correlated with endogenous Txr1 expression. (A) Cell viability, assessed using an MTS assay. Cells were all treated with the same dose of L-OHP $(7.8 \mathrm{mg} / \mathrm{l})$ for $48 \mathrm{~h}$. ${ }^{*} \mathrm{P}<0.05$ and $^{* *} \mathrm{P}<0.01$, SGC-7901 vs. AGS. (B) Txr1 expression in GC cell lines, measured by qPCR, indicating that Txr1 expression is significantly higher in SGC-7901 cells. * $\mathrm{P}<0.05$ vs. the AGS group. (C) Sensitivity of AGS, MNK-45, BGC-823 and SGC-7901 cells to L-OHP was based on their half-maximal inhibitory concentration values $(3.78 \pm 0.32,4.79 \pm 0.21,5.74 \pm 0.17$ and $8.45 \pm 0.10 \mathrm{mg} / 1$, respectively). Results are representative of 3 independent experiments. Error bars represent the standard error of the mean. L-OHP, oxaliplatin; GC, gastric cancer; Txr-1, taxol resistance gene 1.
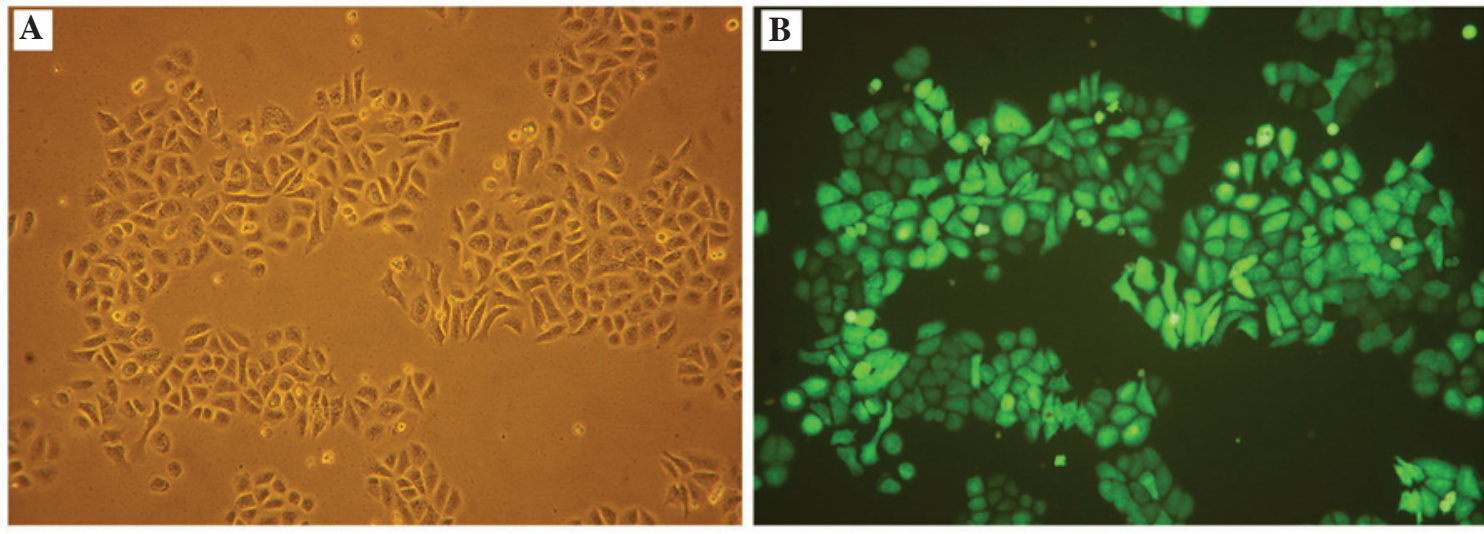

Figure 2. Transduction efficiency in the SGC-7901 cell line following lentivirus-mediated with taxol resistance gene 1 transfection, as detected by (A) light and (B) fluorescence micropscopy. Lentiviral vectors containing green fluorescence protein were expressed in SCG-7901 cells (multiplicity of infection, 100 pfu/cell. Transfection efficiency was $>90 \%$. Magnification, $x 200$.

manufacturer's protocols. Briefly, after a 48-h transfection with the aforementioned treatments, $1 \times 10^{6}$ cells/well were harvested and washed twice with phosphate-buffered saline in 6-well plates. The cells were resuspended in $1 \mathrm{X}$ binding buffer and incubated with Annexin V-FITC and PI for $15 \mathrm{~min}$, in the dark, at room temperature. Apoptosis was detected by FCM using BD CellQuest software (BD Biosciences, Franklin Lakes, NJ, USA).

Statistical analysis. All experiments were performed independently in triplicate. The results are reported as the mean \pm standard deviation, and statistical analyses were performed using the SPSS 17.0 software (SPSS Inc., Chicago, IL, USA). Significant differences were assessed using a standard one-way analysis of variance and two-tailed unpaired Student's t-test. $\mathrm{P}<0.05$ was considered to represent a statistically significant difference.

\section{Results}

L-OHP sensitivity of GC cells is associated with endogenous Txr1 expression. L-OHP sensitivity was detected in the GC cell lines (AGS, MNK-45, BGC-823 and SGC-7901) using an MTS assay; in these cells, viability was inhibited by L-OHP in a dose-dependent manner. The half-maximal inhibitory concentration values in $48 \mathrm{~h}$ of $\mathrm{L}-\mathrm{OHP}$ treatment in the AGS, MNK-45, BGC-823 and SGC-7901 cells were 3.78, $4.79,5.74$ and $8.45 \mathrm{mg} / 1$, respectively. Although the trends were similar, the L-OHP sensitivities of the 4 GC cell lines differed (Fig. 1A), suggesting that other factors may mediate L-OHP sensitivity. Endogenous Txr1 expression in the AGS, MNK-45, BGC-823 and SGC-7901 cells was evaluated using qPCR. As reported in Fig. 1B, Txr1 mRNA levels differed amongst the cell types. Notably, Txr1 levels were significantly higher in the SGC-7901 cells, as compared with the other GC cell lines ( $\mathrm{P}<0.05$ vs. AGS; Fig. 1B), and Txr1 expression varied between the cell lines in a pattern that was converse to the variation observed in L-OHP sensitivity. Furthermore, higher Txr1 expression was correlated with lower L-OHP sensitivity in the SGC-7901 cells, suggesting a negative association between L-OHP sensitivity and endogenous Txr1 expression (Fig. 1C). These data indicated that L-OHP sensitivity is associated with endogenous Txr1 expression in GC cell lines.

Txrl-knockdown sensitizes SGC-7901 cells to L-OHP. Txr1 downregulation was hypothesized to increase L-OHP sensitivity. SGC-7901 cells were selected as an in vitro model, and 


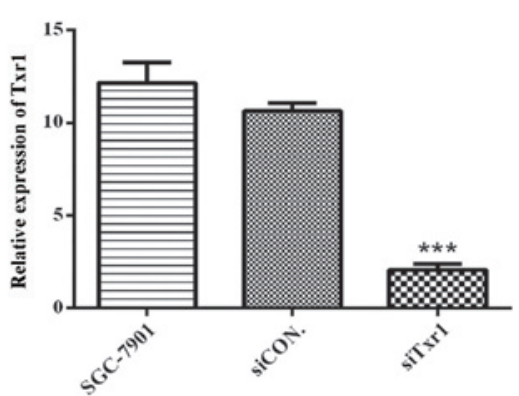

B

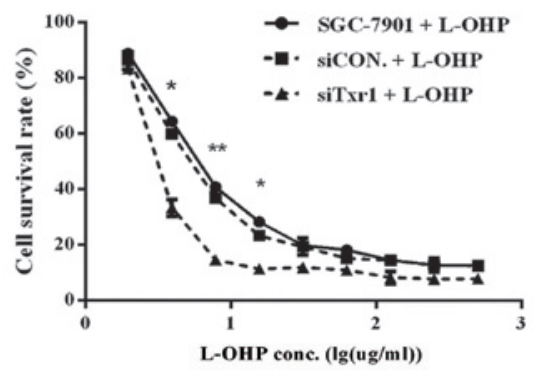

C
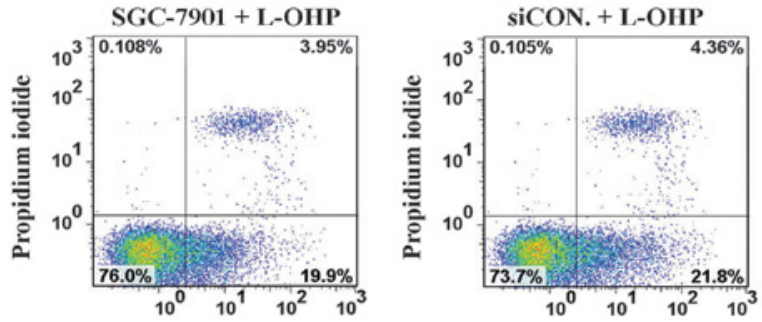

Annexin V-FITC

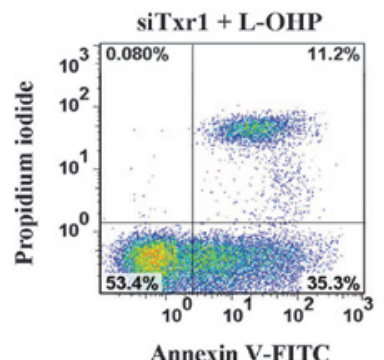

Figure 3. Knockdown of endogenous Txr1 in SGC-7901 cells enhances L-OHP sensitivity. (A) Txr1 expression in SGC-7901 cells following transfection with the siCON or siTxr1 lentivirus, detected using quantitative polymerase chain reaction $\left({ }^{* * *} \mathrm{P}<0.001 \mathrm{vs}\right.$. the control). (B) Viability of SGC-7901 cells following transfection with the siCON or siTxr1 lentivirus and an L-OHP treatment, evaluated using an MTS assay ( ${ }^{*} \mathrm{P}<0.05$ and ${ }^{* *} \mathrm{P}<0.01$ vs. CON). (C) Flow cytometry indicating that the rate of cell apoptosis increased in SGC-7901 cells transfected with the siCON or siTxr1 lentivirus following treatment with L-OHP for $48 \mathrm{~h}$. CON, negative control; L-OHP, oxaliplatin; FITC, fluorescein isothiocyanate; Txr-1, taxol resistance gene 1; si, small interfering.

A

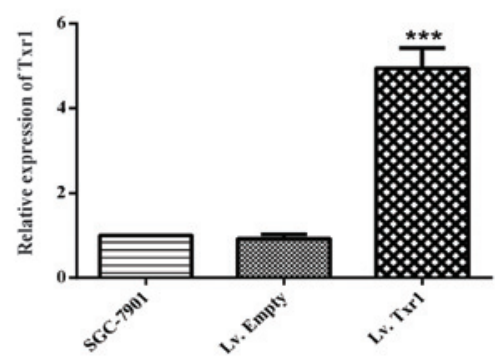

B

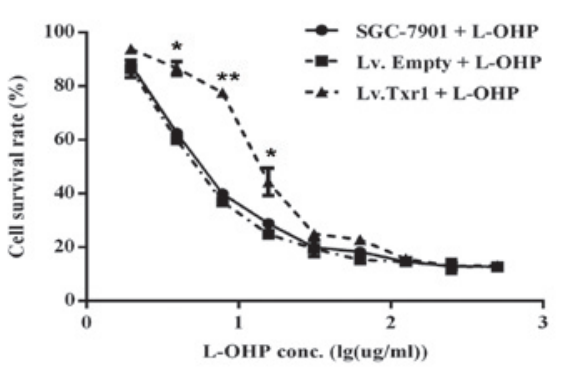

C

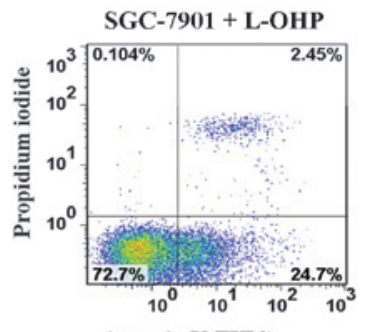

Annexin V-FITC

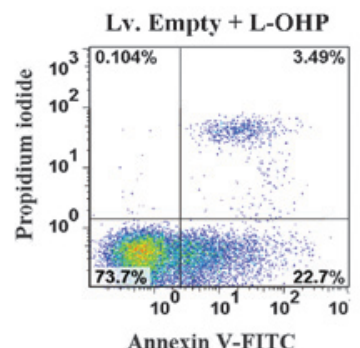

Annexin V-FITC

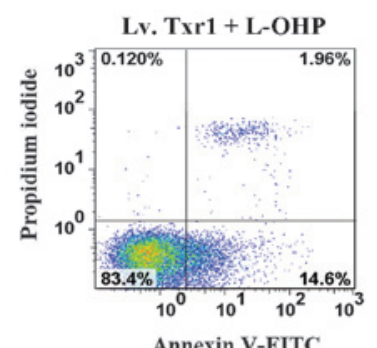

Annexin V-FITC

Figure 4. Txr1 overexpression in SGC-7901 cells enhances resistance to L-OHP. (A) Txr1 expression in SGC-7901 cells following transfection with Lv.Empty or Lv.Txr1, detected using quantitative polymerase chain reaction $\left({ }^{* * *} \mathrm{P}<0.001\right.$ vs. the control). (B) Viability of SGC-7901 cells following Lv.Empty or Lv.Txr1 transfection and L-OHP treatment, evaluated using an MTS assay ( ${ }^{*} \mathrm{P}<0.05$ and ${ }^{* *} \mathrm{P}<0.01$ vs. the control). (C) Flow cytometry, indicating that the cell apoptosis rates decreased in SGC-7901 cells following transfection with Lv.Empty or Lv.Txr1 and treatment with L-OHP for $48 \mathrm{~h}$. Txr-1, taxol resistance gene 1; L-OHP, oxaliplatin; FITC, fluorescein isothiocyanate; Lv, recombinant lentivirus; si, small interfering.

Txr1 was knocked down in these cells by siTxr1 lentiviral transfection. The lentiviral transfection efficiencies in the SGC-7901 cells were evaluated by microscopy $48 \mathrm{~h}$ after transfection, revealing $>90 \%$ lentiviral transfection efficiency (Fig. 2). Txr1 expression was also examined in the SGC-7901 cells following lentiviral transfection using qPCR. Txr1 expression was significantly decreased by $80.5 \%(\mathrm{P}<0.001)$ in siTxr1-transfected cells, as compared with siCON-transfected cells (Fig. 3A), demonstrating that the lentiviral-mediated siRNA effectively and specifically reduced Txrl expression in the SGC-7901 cells. To assess whether decreased Txr1 expression enhanced L-OHP sensitivity, the cells transfected with the siCON or siTxrl lentivirus were treated with L-OHP and viability was measured using an MTS assay. siTxrl-lentiviral transfection enhanced the L-OHP-mediated suppression of SGC-7901 cell viability, particularly at lower 
A

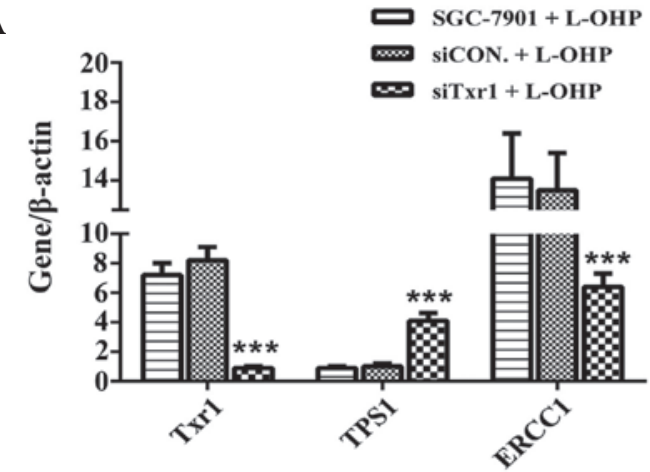

C

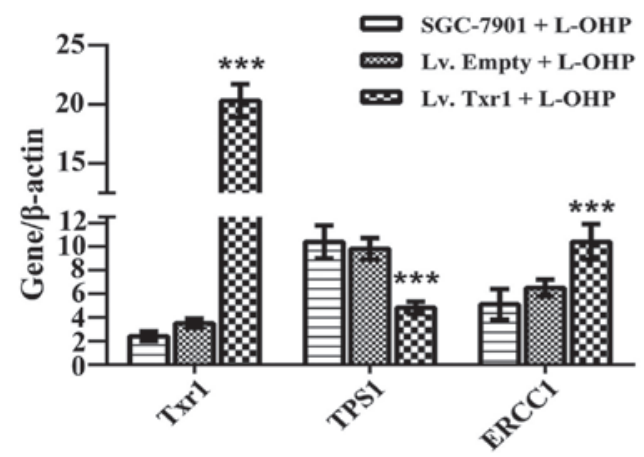

B

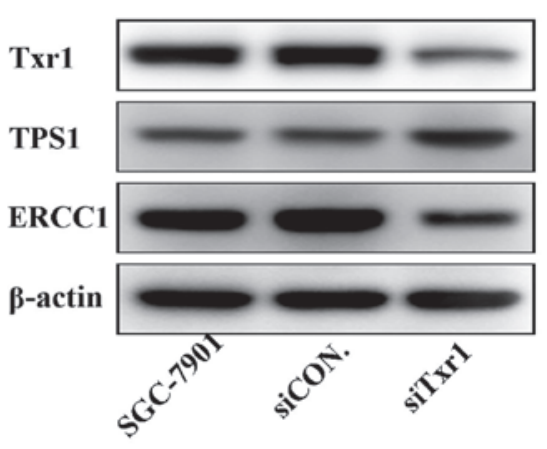

D

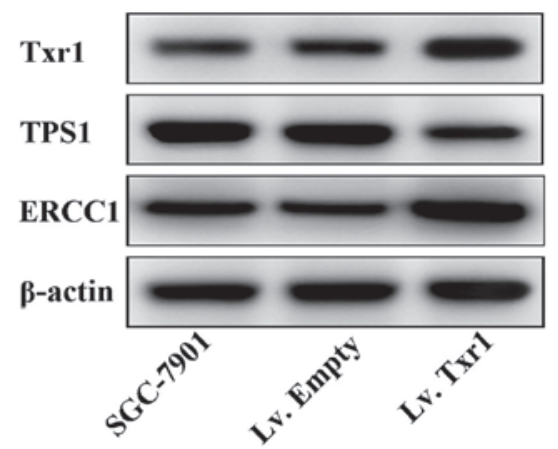

Figure 5. TSP1 and ERCC1 are involved in Txr1-mediated L-OHP sensitivity. (A) Txr1, TSP1 and ERCC1 mRNA levels in SGC7901 cells following siCON or siTxr1 lentiviral transfection and L-OHP treatment, detected using qPCR $\left({ }^{* * * *} \mathrm{P}<0.001\right.$ vs. CON). (B) Txr1, TSP1 and ERCC1 protein levels, examined by western blotting and using $\beta$-actin as a loading control. (C) Txr1, TSP1 and ERCC1 mRNA levels in SGC-7901 cells following control or Txr1 lentiviral transfection and L-OHP treatment, detected by qPCR $\left(^{* * *} \mathrm{P}<0.001\right.$ vs. CON). (D) Txr1, TSP1 and ERCC1 protein levels, examined by western blotting and using $\beta$-actin as a loading control. Txr-1, taxol resistance gene 1; CON, negative control; Lv, recombinant lentivirus; TSP1, thrombospondin-1; ERCC, excision repair cross-complementing 1 protein; L-OHP, oxaliplatin; si, small interfering; qPCR, quantitative polymerase chain reaction.

L-OHP doses (7.8 mg/l) (Fig. 3B). Txr1-knockdown therefore allowed the L-OHP dose used for treatment to be reduced.

Apoptosisis the predominant mechanismofL-OHP-induced toxicity. An Annexin V/PI assay was therefore used to measure the apoptotic frequency in Txr1-knockdown SGC7901 cells treated with L-OHP $(7.8 \mathrm{mg} / \mathrm{l})$. Compared with L-OHP treatment alone, L-OHP treatment with Txrl-knockdown increased the induction of apoptosis in SGC-7901 cells (35.3 vs. 19.9\%, $21.8 \%$ of cells in early apoptosis; and 11.2 vs. $3.95 \%, 4.36 \%$ of cells in late apoptosis) (Fig. 3C). Txr1-knockdown therefore sensitized the cells to L-OHP-induced apoptosis, thereby enhancing the cytotoxic effect of L-OHP.

Txrl overexpression promotes $L-O H P$ resistance in $S G C-7901$ cells. In order to investigate the role of Txr1 in the L-OHP sensitivity of the SGC-7901 cells, Txr1-overexpressing cells were used. Following transfection with Lv.Empty or Lv.Txr1, the SGC-7901 cells were treated with L-OHP at various concentrations (Fig. 4A and B). As expected, Txrl overexpression significantly decreased apoptosis (14.6 vs. $22.7 \%, 24.7 \%$ of cells in early apoptosis; and 1.96 vs. $3.49 \%, 2.54 \%$ of cells in late apoptosis) (Fig. 4C). These gain- and loss-of-function experiments therefore indicated an important function of Txr1 in the L-OHP sensitivity of SGC-7901 cells.

Effects of Txrl on the expression of resistance-associated factors in SGC-7901 GC cells. Due to the inverse correlation observed between Txr1 levels and L-OHP sensitivity, additional mechanistic experiments were warranted. TSP1 and ERCC1 expression was examined by qPCR and western blotting; TSP1 protein levels were significantly increased and ERCC1 levels were decreased in the siTxr1-transfected group compared with the CON groups $(\mathrm{P}<0.001$; Fig. $5 \mathrm{~A}$ and $\mathrm{B})$. Whereas, in the lentivirus-mediated overexpression Txr1 group, TSP1 protein levels were decreased and ERCC1 levels were increased, as compared with the CON groups $(\mathrm{P}<0.001$; Fig. 5C and D). These data suggest that Txrl-knockdown may mediate GC sensitization to L-OHP via TSP1 and ERCC1.

\section{Discussion}

Surgical resection for GC is the only potentially curative treatment method, however, the majority of patients relapse following resection; therefore, combinatorial treatment approaches are standard for disease that is advanced beyond stage 1B (12). A previous, large meta-analysis of adjuvant chemotherapy in GC conducted at the individual patient level confirmed a $6 \%$ improvement in outcomes following 5 -fluorouracil (5-FU)-based chemotherapy compared with surgery alone (hazard ratio, 0.82 ; 95\% confidence interval, 0.76-0.90; $\mathrm{P}<0.001)$ in all subgroups tested (13). Combination regimens based on a platinum-fluoropyrimidine doublet are typically used as first-line chemotherapy options (14). L-OHP has been a crucial component in combination therapies since its clinical introduction, resulting in modest survival improvements across multiple malignancies (15). The Capecitabine and Oxaliplatin Adjuvant Study in Stomach Cancer trial (16), conducted in South Korea, China and Taiwan, evaluated an adjuvant chemotherapy combining capecitabine 
with L-OHP following curative D2 gastrectomy and compared results with surgery alone, reporting significantly improved OS and disease-free survival.

L-OHP forms the basis of multiple treatment regimens. Despite its modest activity as a single agent, L-OHP exerts significant activity when used in combination with other drugs (17). As with other anticancer drugs, tumor cells can acquire resistance to the cytotoxic effects of L-OHP. The mechanism of L-OHP resistance has previously been demonstrated to be mediated by Txr1, which downregulates TSP1 in tumors $(11,18)$. In our previous study, we reported that the 5-year OS rate of GC patients with high Txrl expression was lower than that of patients with low Txr1 expression; Txr1 expression may therefore be used as an independent prognostic indicator (11).

To assess the role of Txr1 in the L-OHP resistance of GC cells, the present study first examined Txr1 expression in 4 GC cell lines and revealed a negative association between L-OHP sensitivity and endogenous Txr1 expression. Following transfection with siTxr1, the SGC-7901 cells demonstrated increased L-OHP sensitivity, indicating that endogenous Txr1 may protect cells from L-OHP cytotoxicity. To demonstrate that Txr1 conferred L-OHP resistance, cells overexpressing exogenous Txr1 using Lv.Txr1 were used; these cells exhibited increased L-OHP resistance. A likely association between Txr1 expression and L-OHP resistance was therefore identified.

TSP1 gene expression was also analyzed in the present study, revealing that TSP1 levels were positively correlated with L-OHP sensitivity. TSP1 was previously reported to have anti-angiogenic and pro-apoptotic activities in oncogenesis (19). TSP1 is an important mediator of taxane-induced apoptosis via the prevention of angiogenesis and the induction of apoptosis in malignant cells (20).

Papadaki et al (9) reported that, amongst lung adenocarcinoma patients administered a combinatorial treatment of docetaxel and gemcitabine, patients demonstrating low Txr1 and high TSP1 expression levels experienced higher survival rates compared with patients demonstrating the converse. Similar results were obtained in non-small cell lung cancer patients treated with docetaxel in association with cisplatin or gemcitabine (21). Txrl is therefore likely to have diverse effects on different cell types in a TSP1-dependent manner. An inverse correlation has previously been identified between TSP1 mRNA levels and the gastric cardia adenocarcinoma tumor-node-metastasis stage (22). In our previous study, exogenous Txr1 expression was associated with decreased TSP1 mRNA in BGC-823 cells, possibly contributing to taxol resistance (11). In the present study, Txr1-knockdown in SGC-7901 cells led an to increased TSP1 expression level, apoptosis rate and L-OHP sensitivity. Txr1 overexpression decreased TSP1 expression, resulting in apoptosis inhibition, which may be the underlying etiology of L-OHP resistance in GC.

DNA repair is a principal mechanism underlying the resistance to platinum-based therapy (23). The repair of platinum-associated DNA damage requires resolution of the associated DNA break, which involves a variety of repair proteins, including ERCC1, which may have clinical potential as a predictive biomarker (24). In the present study, ERCC1 expression was increased when Txr1 was exogenously expressed, meaning that Txr1 and ERCC1 mRNA levels are negatively correlated with L-OHP sensitivity. Several pre-clinical studies have demonstrated that ERCC1 has an important role in determining platinum drug sensitivity; increased ERCC1 expression is associated with platinum drug resistance (25-27). Clinically, high ERCC1 levels are correlated with poor responses to platinum-based chemotherapy in GC (28). ERCC1 acts on larger lesions covering 20-25 nucleotides, therefore, an efficient DNA repair capacity of ERCC1 appears to be critical for resistance to platinum drugs $(29,30)$. Previous data from in vitro systems have revealed that suppression of ERCC1 expression enhances or restores the sensitivity to platinum. This has significant clinical implications, as the inclusion of factors targeting ERCC1 may increase platinum activity and/or oppose resistance, although this remains unreported in patients (31).

Drug resistance in GC patients appears to be complex. The presence of one protein can markedly affect a multitude of proteins and pathways, and the associations between apparently unrelated proteins and pathways are only now being revealed (32). The screening and validating of molecular biomarkers capable of predicting the response to different chemotherapeutic agents represent significant steps toward personalized treatment for cancer patients. The present study focused on Txr1, as this gene has previously been identified as a drug resistance gene in numerous solid tumors, including GC. The current study demonstrated that Txr1-knockdown in SGC7901 cells enhanced their chemosensitivity to L-OHP. The identification of Txr1 as a potential resistance gene in L-OHP therapy provides a fundamental basis for novel approaches in developing L-OHP-based therapeutic strategies. Furthermore, the introduction of novel anti-microtubule platinum agents requires elucidation of the biological mechanisms mediating the resistance effects of these drugs.

\section{Acknowledgements}

The present study was supported by the National Natural Science Foundation of China (grant no. 81172317) and Beijing Municipal Administration of Hospitals Clinical Medicine Development of Special Funding Support (grant no. ZYLC201504).

\section{References}

1. Ferlay J, Soerjomataram I, Dikshit R, Eser S, Mathers C, Rebelo M, Parkin DM, Forman D and Bray F: Cancer incidence and mortality worldwide: Sources, methods and major patterns in GLOBOCAN 2012. Int J Cancer 136: E359-E386, 2015.

2. Foo M and Leong T: Adjuvant therapy for gastric cancer: Current and future directions. World J Gastroenterol 20: 13718-13727, 2014.

3. Park SC and Chun HJ: Chemotherapy for advanced gastric cancer: Review and update of current practices. Gut Liver 7: 385-393, 2013.

4. Zhao Q, Zhang H, Li Y, Liu J, Hu X and Fan L: Anti-tumor effects of CIK combined with oxaliplatin in human oxaliplatin-resistant gastric cancer cells in vivo and in vitro. J Exp Clin Cancer Res 29: 118, 2010.

5. Rabik CA and Dolan ME: Molecular mechanisms of resistance and toxicity associated with platinating agents. Cancer Treat Rev 33: 9-23, 2007.

6. Wang J, Zhou XQ, Li JY, Cheng JF, Zeng XN, Li X and Liu P: Prognostic significance of ERCC1 expression in postoperative patients with gastric cancer. Chin J Cancer Res 26: 323-330, 2014. 
7. Yao A, Wang Y, Peng X, Ye R, Wang Q, Qi Y and Zhou F: Predictive value of excision repair cross-complementation group 1 expression for platinum-based chemotherapy and survival in gastric cancer: A meta-analysis. J Cancer Res Clin Oncol 140: 2107-2117, 2014.

8. Lih CJ, Wei W and Cohen SN: Txr1: A transcriptional regulator of thrombospondin-1 that modulates cellular sensitivity to taxanes. Genes Dev 20: 2082-2095, 2006.

9. Papadaki C, Mavroudis D, Trypaki M, Koutsopoulos A, Stathopoulos E, Hatzidaki D, Tsakalakil E, Georgoulias V and Souglakos J: Tumoral expression of TXR1 and TSP1 predicts overall survival of patients with lung adenocarcinoma treated with first-line docetaxel-gemcitabine regimen. Clin Cancer Res 15: 3827-3833, 2009.

10. Bai Z, Zhang Z, Qu X, Han W and Ma X: Sensitization of breast cancer cells to taxol by inhibition of taxol resistance gene 1 . Oncol Lett 3: 135-140, 2012.

11. Bai ZG, Qu X, Han W, Ma XM, Zhao XM and Zhang ZT: Expression of taxol resistance gene 1 correlates with gastric cancer patient clinical outcome and induces taxol resistance. Mol Med Rep 3: 1071-1078, 2010.

12. Waddell T, Verheij M, Allum W, Cunningham D, Cervantes A and Arnold D; European Society for Medical Oncology (ESMO); European Society of Surgical Oncology (ESSO); European Society of Radiotherapy and Oncology (ESTRO): ESMO; ESSO; ESTRO: Gastric cancer: ESMO-ESSO-ESTRO clinical practice guidelines for diagnosis, treatment and follow-up. J Eur J Surg Oncol 40: 584-591, 2014.

13. GASTRIC (Global Advanced/Adjuvant Stomach Tumor Research International Collaboration) Group; Paoletti X, Oba K, Burzykowski T, Michiels S, Ohashi Y, Pignon JP, Rougier P, Sakamoto J, Sargent D, Sasako M, et al: Benefit of adjuvant chemotherapy for resectable gastric cancer: A meta-analysis. JAMA 303: 1729-1737, 2010.

14. Waddell T, Verheij M, Allum W, Cunningham D, Cervantes A and Arnold D; European Society for Medical Oncology (ESMO); European Society of Surgical Oncology (ESSO); and European Society of Radiotherapy and Oncology (ESTRO): Gastric cancer: ESMO-ESSO-ESTRO Clinical Practice Guidelines for diagnosis, treatment and follow-up. Ann Oncol 24 (Suppl 6) vi57-vi63, 2013

15. Alian OM, Azmi AS and Mohammad RM: Network insights on oxaliplatin anti-cancer mechanisms. Clin Transl Med 1: 26, 2012.

16. Noh SH, Park SR, Yang HK, Chung HC, Chung IJ, Kim SW $\mathrm{Kim} \mathrm{HH}$, Choi JH, Kim HK, Yu W, et al; CLASSIC trial investigators: Adjuvant capecitabine plus oxaliplatin for gastric cancer after D2 gastrectomy (CLASSIC): 5-year follow-up of an open-label, randomised phase 3 trial. Lancet Oncol 15: 1389-1396, 2014.

17. Di Francia R, Siesto RS, Valente D, Del Buono A, Pugliese S, Cecere S, Cavaliere C, Nasti G, Facchini G and Berretta M Current strategies to minimize toxicity of oxaliplatin: Selection of pharmacogenomic panel tests. Anticancer Drugs 24: 1069-1078, 2013.

18. Bi J, Bai Z, Ma X, Song J, Guo Y, Zhao J, Yi X, Han S and Zhang Z: Txr1: An important factor in oxaliplatin resistance in gastric cancer. Med Oncol 31: 807, 2014
19. Sid B, Sartelet H, Bellon G, El Btaouri H, Rath G, Delorme N, Haye B and Martiny L: Thrombospondin 1: A multifunctional protein implicated in the regulation of tumor growth. Crit Rev Oncol Hematol 49: 245-258, 2004

20. van Amerongen R and Berns A: TXR1-mediated thrombospondin repression: A novel mechanism of resistance to taxanes? Genes Dev 20: 1975-1981, 2006.

21. Papadaki C, Tsaroucha E, Kaklamanis L, Lagoudaki E, Trypaki M, Tryfonidis K, Mavroudis D, Stathopoulos E, Georgoulias V and Souglakos J: Correlation of BRCA1, TXR1 and TSP1 mRNA expression with treatment outcome to docetaxel-based first-line chemotherapy in patients with advanced/metastatic non-small-cell lung cancer. Br J Cancer 104: 316-323, 2011

22. Guo W, Dong Z, He M, Guo Y, Guo J, Chen Z, Yang Z and Kuang G: Aberrant methylation of thrombospondin-1 and its association with reduced expression in gastric cardia adenocarcinoma. J Biomed Biotechnol 2010: 721485, 2010.

23. Reardon JT, Vaisman A, Chaney SG and Sancar A: Efficient nucleotide excision repair of cisplatin, oxaliplatin, and Bis-aceto-ammine-dichloro-cyclohexylamine-platinum (IV) (JM216) platinum intrastrand DNA diadducts. Cancer Res 59: 3968-3971, 1999.

24. Deng Q, Yang H, Lin Y, Qiu Y, Gu X, He P, Zhao M, Wang H, $\mathrm{Xu} Y$, Lin Y, et al: Prognostic value of ERCC1 mRNA expression in non-small cell lung cancer, breast cancer, and gastric cancer in patients from Southern China. Int J Clin Exp Pathol 7: 8312-8321, 2014.

25. Lee KB, Parker RJ, Bohr V, Cornelison T and Reed E: Cisplatin sensitivity/resistance in UV repair-deficient Chinese hamster ovary cells of complementation groups 1 and 3. Carcinogenesis 14: 2177-2180, 1993.

26. Melton DW, Ketchen AM, Núñez F, Bonatti-Abbondandolo S, Abbondandolo A, Squires S and Johnson RT: Cells from ERCC1-deficient mice show increased genome instability and a reduced frequency of S-phase-dependent illegitimate chromosome exchange but a normal frequency of homologous recombination. J Cell Sci 111: 395-404, 1998.

27. Youn CK, Kim MH, Cho HJ, Kim HB, Chang IY, Chung MH and You HJ: Oncogenic H-Ras up-regulates expression of ERCC1 to protect cells from platinum-based anticancer agents. Cancer Res 64: 4849-4857, 2004

28. Metzger R, Leichman CG, Danenberg KD, Danenberg PV, Lenz HJ, Hayashi K, Groshen S, Salonga D, Cohen H, Laine L, et al: ERCC1 mRNA levels complement thymidylate synthase mRNA levels in predicting response and survival for gastric cancer patients receiving combination cisplatin and fluorouracil chemotherapy. J Clin Oncol 16: 309-316, 1998

29. Reed E: Platinum-DNA adduct, nucleotide excision repair and platinum based anti-cancer chemotherapy. Cancer Treat Rev 24: 331-344, 1998.

30. Wood RD, Mitchell M, Sgouros J and Lindahl T: Human DNA repair genes. Science 291: 1284-1289, 2001.

31. Reed E, Yu JJ, Davies A, Gannon J and Armentrout SL: Clear cell tumors have higher mRNA levels of ERCC1 and XPB than other histological types of epithelial ovarian cancer. Clin Cancer Res 9: 5299-5305, 2003

32. Vogelstein B and Kinzler KW: Cancer genes and the pathways they control. Nat Med 10: 789-799, 2004 